\title{
1 An in-vivo digital atlas of the spatially 2 reliable cerebral vasculature in mice
}

3 Rukun Hinz ${ }^{l}$, Meriam Malekzadeh ${ }^{l}$, Lore M. Peeters ${ }^{l}$, Verdi Vanreusel ${ }^{l}$, Marleen Verhoye ${ }^{l}$,

$4 \quad$ Annemie Van der Linden ${ }^{1}$, Georgios A. Keliris ${ }^{1}$

$6 \quad{ }^{1}$ Bio-Imaging Lab, University of Antwerp, Belgium

7

8

9 Prof. Georgios Keliris

10 Bio-imaging lab, University of Antwerp

11 Campus Drie Eiken- Building Uc 1.07

12 Universiteitsplein 1 -2610 Wilrijk - Belgium

13 Tel. +32322652772

14 Email: Georgios.Keliris@uantwerpen.be

Keywords: Digital cerebral vascular atlas, Mouse, Resting-state functional MRI, Vascular

18 skeleton, Magnetic Resonance Imaging, Angiography, TOF MRA 


\section{Abstract}

20 Cerebral vascular abnormalities can have a large impact on brain function and have been

21 frequently detected as a comorbidity in various neuropathologies. The mouse is the most

22 common pre-clinical animal model used to investigate neuropathologies and thus cerebral

23 vascular atlases of this species are indispensable. In particular, an atlas derived from multiple

24 animals and that can address inter-subject variability is still absent. The current study presents

25 a mouse cerebral vascular atlas developed from MR 2D-TOF angiograms acquired from a

26 relatively high number $(\mathrm{N}=21)$ of 12 weeks old male $\mathrm{C} 57 \mathrm{BL} / 6$ mice. The vascular atlas we

27 present depicts large arteries, veins and sinuses that are more spatially reliable across animals

28 than smaller vessels. The atlas is available for download and contains multiple datasets: i) a

29 cerebral vascular atlas; ii) a digital segmentation of large vessels (arteries and veins); iii) a

30 high resolution T2 anatomical background aligned to the atlas; iv) a skeletonized vascular

31 atlas; and v) a vascular graph model. We suggest that these components provide a very potent

32 analysis tool for mouse fMRI data and that the atlas can be used as a template for multimodal

33 brain imaging studies. To this end, we also demonstrate an explicit application of the atlas

34 for the investigation of vascular influences in resting-state fMRI. 
35

\section{Introduction}

The cerebral vasculature has an indispensable supporting role in maintaining the energy homeostasis of the brain by supplying it with oxygen and nutrients as well as clearing the brain of waste metabolites. $[1,2]$. Any structural aberrations in the cerebral vasculature, such as those due to stroke, can deplete the continuous blood supply and can result in cell death and loss of function. Furthermore, structural vascular abnormalities have been shown to commonly arise as comorbidities in various neuropathological disorders, such as Alzheimer's disease, Huntington's disease and multiple sclerosis [3-5]. Hence, appropriate sensitive tools to investigate cerebral vasculature are required.

In preclinical research, mice models are by far the most commonly used animal models to study neuropathological disorders. During the last decades, technological advancements have been made in preclinical imaging allowing for the characterization of the mouse brain's vasculature at different spatial scales. For example, ex-vivo imaging techniques like knifeedge scanning microscopy (KESM), micro-optical sectioning tomography (MOST), serial two photon tomography and light sheet microscopic (LSM) imaging have been used to acquire high-resolution imaging data of the vascular architecture up to the capillary level [611]. These techniques result in the acquisition of large datasets that allow in-depth analysis of the whole brain vasculature. Furthermore, in-vivo imaging techniques, such as computed tomography (CT), magnetic resonance angiography (MRA) and transcranial functional ultrasound (fUS) have been optimized to visualize cerebral vasculature with its main advantage that it can follow-up vascular integrity in the same subject over time [12-14]. These latter techniques predominantly image the macro vasculature, but CT and MR imaging 
57 can also be performed ex-vivo with longer acquisitions and higher resolution to acquire

58 information about the microvasculature $[15,16]$. Using the aforementioned techniques,

59 several vascular atlases have been developed to assist in identifying the mouse brain

60 vasculature. In one study, ex-vivo CT imaging was combined with MRI to image the mouse

61 whole brain vasculature of four mice together with an anatomical background [17]. This atlas

62 provides the segmentation of the vasculature at the macroscopic level allowing the

63 identification of most large vessels. A more detailed vascular atlas was obtained in the study

64 of Xiong et al. using micro-optical sectioning tomography (MOST) [18]. In that study, the

65 entire mouse brain vascular architecture was mapped of five animals and up to the capillary

66 level allowing the identification of the whole brain vasculature as well as the investigation

67 of microvascular densities in the brain.

68 Knowledge of the vascular architecture is not only important for identifying vascular

69 alterations in disease states but can also play a key role in supporting a variety of

70 neuroimaging techniques. Imaging studies such as fUS and intrinsic optical imaging (IOS)

71 are exclusively based on acquiring vascular information, but with limited spatial resolution

$72[19,20]$. Vascular atlases that would be suitable to use across studies and incorporate

73 anatomical information can help to identify the spatial location of the vessel. Furthermore,

74 vessel occlusion studies that investigate stroke can benefit from vascular atlases to select the

75 appropriate vessel to occlude or to estimate the size of the hypoxic area. An important number

76 of studies that can benefit from vascular atlases are those using functional MRI (fMRI) and/or

77 resting state fMRI (rsfMRI). These techniques rely on the acquisition of blood oxygenation

78 level dependent (BOLD) signals that are mainly derived from the hemodynamic responses 
caused by the neurovascular coupling [21]. However, studies have shown that other vascular effects, such as vascular drainage, also influence the BOLD signal [22, 23]. Additionally, resting state signals have been shown to be confounded by signals from large arteries and veins [24]. These confounding signals are thought to be systemically produced from outside the brain and seem to be propagating throughout the blood vessels [25]. A vascular atlas that can be readily used in such studies, can help in identifying potential vascular confounds and assist in further analysis steps aiming to remove them.

To be able to apply a vascular atlas in the aforementioned studies, it is assumed that the spatial location of the vasculature is conserved between subjects. However, the study of Xiong et al. has shown that the spatial location of small vessels $(<40 \mu \mathrm{m})$ can be highly variable between subjects of the same strain, while larger vessels $(>40 \mu \mathrm{m})$ are more spatially reliable [18]. An efficient method to visualize spatially reliable vessels is to develop a vascular atlas based on angiograms from multiple subjects. As such, spatial unreliable vessels will be suppressed by averaging the datasets. However, mouse vascular atlases to date focused on the visualization of the detailed vasculature in a single mouse or a small number of subjects and were created ex-vivo in order to use techniques that allow high resolution [18, 26]. Thus, existing mouse vascular atlases are less suitable for application across studies.

In this study, we developed and present an in-vivo acquired cerebral vascular atlas of the large vessels using 21 male C57BL/6 mice of 12 weeks of age. To this end, non-invasive imaging, 2D time of flight (TOF) MRA was used and combined with high-resolution anatomical T2-weighted MRI for spatial identification. Our digital atlas is provided together with an anatomical MRI template, and thus allows easy and direct co-registration to MRI 
101 datasets or can be used as a reference template for segmenting newly acquired individual

102 angiography. We also provide a skeletonized version of the atlas that can be used as a graph

103 to calculate parameters such as path length. Moreover, in this study, we demonstrate an

104 explicit application of the atlas for the investigation of vascular influences in resting-state

105 fMRI by estimating the overlap between vasculature and resting state networks (RSNs).

106

107

108

109

110

111

112

113

114

115

116

117

118

119

120

\section{Material and Methods}

\subsection{Animals and ethical statement}

The study was conducted using 12 weeks old male C57BL/6 wild-type mice $(\mathrm{N}=21)$ (Janvier). Mice were kept under a day/night cycle of $12 \mathrm{~h} / 12 \mathrm{~h}$ respectively, with an average room temperature of $20-24^{\circ} \mathrm{C}$ and $40 \%$ humidity. All mice were group housed with ad libitum access to water and standard rodent chow. All applicable institutional and/or national guidelines for the care and use of animals were followed. Procedures were performed in accordance with the European Directive 2010/63/EU on the protection of animals used for scientific purposes. The protocols were approved by the Committee on Animal Care and Use at the University of Antwerp, Belgium (permit number: 2016-10) and all efforts were made to minimize animal suffering. Experiments have been reported in compliance with the ARRIVE guidelines.

\subsection{Data availability statement}

Data used in this study are available online and can be downloaded at www.uantwerpen.be/en/research-groups/bio-imaging-lab/research/mri-atlases/. Datasets 
121 include 2D-TOF MRA of each individual subject, vascular atlas, skeletonized version of the

122 vascular atlas and the anatomical study-specific template.

\subsection{Imaging procedure}

124 All MR imaging procedures were performed on a 9.4 T Biospec MR system (Bruker, 125 Germany) with a receive-only 4 channel array cryoprobe (Bruker, Germany) combined with 126 a volume transmit resonator. All animals underwent MRA imaging, whereas a partial group $127(\mathrm{~N}=13)$ was scanned a second time to extract rsfMRI data.

\subsubsection{MRA imaging}

129 For MRA imaging, animals were first anesthetized using 5\% isoflurane in a gas mixture of $13030 \% \mathrm{O} 2$ and $70 \% \mathrm{~N} 2$ for induction, which was then subsequently decreased to $2 \%$ isoflurane

131 for maintenance during the remainder of the session. All mice were placed head-fixed in the 132 scanner by using ear- and tooth-bars and ophthalmic ointment was applied to the eyes to 133 prevent desiccation. During the experimental procedure, animals' physiology was closely 134 monitored. As such, respiratory rate was followed using a pressure sensitive pad (MR135 compatible Small Animal Monitoring and Gating system, SA Instruments, Inc.). Body 136 temperature was followed using a rectal thermistor and was maintained at $(37.0 \pm 0.1)^{\circ} \mathrm{C}$ 137 using a feedback controlled warm air heat system (MR-compatible Small Animal Heating 138 System, SA Instruments, Inc.). Additionally, blood oxygenation was monitored using a pulse 139 oxygenation meter (MR-compatible Small Animal Monitoring and Gating system, SA 140 Instruments, Inc.). 
141 MRI data acquisition consisted out of the measurement of three orthogonal oriented 2D-TOF-

142 MRA to assess the macrovasculature with blood flow in each direction: (FOV: $(20 \times 20) \mathrm{mm}^{2}$,

143 matrix dimension (MD: [320x320], 110, 130, 90 slices respectively in the coronal, axial and

144 sagittal oriented scans, slice thickness (ST): 0,3 mm, slice shift: $0.15 \mathrm{~mm}$, voxel dimension

$145(0.0625 \times 0.0625 \times 0.15) \mathrm{mm}^{3}$, TE/TR: 3.2/17 ms, flip angle: $60^{\circ}$,number of averages: 3$)$.

146 Subsequently, a high-resolution 3D T2-weighted Turbo-RARE was acquired for the

147 development of an anatomical template (FOV: (20x20x15) mm², MD: [256x256x192], voxel

148 dimension $=(0.078 \mathrm{~mm})^{3}$, TE/TR: 40/2500 ms, RAREfactor: 16). After the imaging

149 procedure, animals were kept in a recovery box with infrared heating until they were fully

150 awake to compensate for post-anaesthethic decreased body temperature.

\subsection{2. $\quad$ Resting state functional MRI}

152

153

154

155

156

157

158

159

160

161

162
RsfMRI was acquired 1 to 2 weeks after the MRA imaging session. To do so, animals' anesthesia was first induced with 5\% isoflurane in a gas mixture of $30 \% \mathrm{O}_{2}$ and $70 \% \mathrm{~N}_{2}$ and was lowered to $2 \%$ isoflurane for maintenance. Mice were head-fixed and were monitored similarly as for the previous MRA session. Ophthalmic ointment was applied to the eyes. After fixation, animals received a subcutaneous bolus of medetomidine $(0.05 \mathrm{mg} / \mathrm{kg})$ which was 15 minutes later followed by a subcutaneous continuous infusion of medetomidine $(0.1$ $\mathrm{mg} / \mathrm{kg} / \mathrm{h}$ ). After bolus injection, isoflurane concentration was gradually decreased to $0.4 \%$ isoflurane over a time period of 5 minutes.

In each animal, three orthogonally placed T2-weighted Turbo-RARE (FOV: (20x20) mm², MD: [256x256], 12 slices, ST: 0.4 mm, TE/TR: 33/2500 ms, RAREfactor: 8) anatomical images were acquired to ensure slice position uniformity between animals. Next, a coronal 
163 oriented T2-weighted anatomical reference scan was acquired using a Turbo-RARE

164 sequence (FOV: $(24 \times 16) \mathrm{mm}^{2}, \mathrm{MD}:$ [256x256], 16 slices , ST: $0.5 \mathrm{~mm}, \mathrm{TE} / \mathrm{TR}: 33 / 2500 \mathrm{~ms}$,

165 RAREfactor: 8) to be used as an anatomical background for functional imaging data.

166 Afterwards, B0 field maps were acquired to estimate magnetic field inhomogeneity that was

167 used for the subsequent local shimming procedure within a rectangular volume of interest

168 covering the mouse brain. Thirty minutes post-bolus injection, rsfMRI scans were acquired

169 using a T2*-weighted single shot echo planar imaging (EPI) sequence (FOV: $(24 \times 16) \mathrm{mm}^{2}$,

170 MD: [96x64], 16 slices, ST: $0.5 \mathrm{~mm}$, voxel dimension: $(0.25 \times 0.25 \times 0.50) \mathrm{mm}^{3}$, TE/TR:

$17116 / 1000 \mathrm{~ms}, 600$ volumes). After the imaging procedure, mice received a subcutaneous bolus

172 injection of $0.1 \mathrm{mg} / \mathrm{kg}$ atipamezole (Antisedan, Pfizer, Karlsruhe, Germany) to counteract

173 the effects of medetomidine and then the animals were placed in a recovery box with infrared

174 heating until they were fully awake.

175

176

177

178

179

180

181

182

\subsection{Data Processing}

\subsubsection{Anatomical Template}

The 3D-T2 weighted anatomical scans of each subject were used to develop a study-specific anatomical template. All co-registration processing was performed using Advanced Normalization Tools (ANTS). Scans were first debiased to equalize signal intensities over the FOV. Afterwards, the template was created using the standard ANTS create template protocol.

\subsubsection{MRA}


183 The 2D-TOF MRA sequence is an excellent tool for non-invasive imaging of the complete

184 mouse brain vasculature. This sequence allows for the detection of vascular flow 185 perpendicular towards the slice direction. Therefore, three orthogonally oriented 2D-TOF 186 MRAs were used to visualize vascular flow in the rostral-caudal (fig.1A), dorsal-ventral

187 (fig.1B) and left-right (fig.1C) direction. To develop subject specific angiograms, scans were

188 first visually inspected for motion artifacts and then combined using a maximum Intensity

189 Projection (MIP) (fig.1D). Afterwards, each subject vascular angiogram was co-registered to

190 the anatomical template by using the previously estimated subject to template transformation

191 parameters from the template creation step. Finally, co-registered vascular angiograms of

192 each subject were averaged across all subjects to create a vascular atlas.

193 Next, processing of the data was performed in Amira 5.4 (FEI, Thermo Scientific). Individual

194 blood vessels were manually delineated on the vascular atlas and labeling was based on 195 previous reported vascular atlases $[17,18]$. Afterwards, the vascular atlas was skeletonized 196 using Amira 5.4 skeletonization tool transforming the developed atlas into a nodes and edges 197 model. This model was then saved as centerline voxels in Amira 5.4 which converts the nodes 198 and edges model into single voxel lines representing the inner core of the vessels. 199 Additionally, the nodes and edges model allowed for automatic clustering of enclosed 200 circuits. As the circuits of large arteries and veins did not overlap, the nodes and edges model 201 facilitated the automatic separation of arteries and veins. Information coming from the nodes 202 and edges model was then used to develop a vascular graph model in Matlab code (MATLAB 203 R2019a, The MathWorks Inc. Natick, MA, USA). 


\subsubsection{Resting state functional MRI}

206

207

208

209

210

211

212

213

214

215

216

217

218

219

220

221

222

223

224

225

226

Data processing was performed using SPM 12 (Statistical Parametric Mapping, http://www.fil.ion.ucl.ac.uk), Gift toolbox (Group ICA of fMRI toolbox version 3.0a: http://icatb.sourceforge.net) and Matlab 2014a (MATLAB R2014a, The MathWorks Inc. Natick, MA, USA). Functional data was first realigned using a 6-parameter (rigid body) spatial transformation. Next, images were used to create a study-specific EPI template by normalizing the first volume EPI of each subject to the first volume of a single subject by using a global 12-parameter affine transformation followed by a non-linear transformation and an averaging of all normalized scans. Afterwards, individual subject functional datasets were normalized to this study-specific EPI template using a global 12-parameter affine transformation and subsequently a non-linear transformation. Normalized data was then smoothed in-plane by using a Gaussian kernel with a full-width half-maximum of twice the voxel size $(0.5 \times 0.5 \mathrm{~mm})$. Data were then further band pass filtered between $0.01-0.15 \mathrm{~Hz}$.

To extract RSN from rsfMRI data, an independent component analysis (ICA) was performed with the Infomax algorithm with 15 pre-defined number of components. Components representing RSN were calculated by performing a one-sample t-test to obtain distinct networks and identified by comparison to previously observed RSNs [27-30]. ICA data was then co-registered from the EPI template space to the vascular atlas high-resolution 3D-T2 weighted anatomical template space by performing a rigid, affine and non-linear transformation using the ANTS toolbox. For this transformation, each voxel index from the lower resolution EPI data was used as a value instead of a binary mask so that we could calculate overlap with the original EPI voxels after nearest neighbor interpolation. To identify 
227 RSNs with potential vascular influences, RSNs were sorted based on the percentage overlap

228 with the vascular atlas. To this end, for each RSN, the vascular overlap fraction (VOF) was

229 calculated by dividing the total amount of (unique) voxels overlapping with the vascular

230 skeleton by the total amount of unique voxels of that RSN.

$$
V O F=\frac{\# \text { voxels vascular skeleton within } R S N}{\# \text { voxels } R S N}
$$

232 Similarly, we calculated partial VOF for specific vascular compartments (i.e. arterial, 233 venous/sinous) or specific vessels by replacing the total vascular skeleton with the skeleton 234 of the vascular compartment of interest

$$
p V O F=\frac{\# \text { voxels specific vascular skeleton within } R S N}{\# \text { voxels } R S N}
$$

236 After sorting, the two RSNs with the highest vascular overlap were selected and further 237 analyzed in order to identify the specific arteries and vessels overlapping with these RSNs.

238 To this end, the percentage of vessel inclusion (PVI) within each network was calculated for each artery and vein by dividing the number of voxels of each RSN overlapping with each

240 vessel's skeleton with the number of voxels of that vessel's skeleton and converting to 241 percentage.

$$
P V I=\frac{\# \text { voxel RSN within vessel skeleton }}{\# \text { voxels vessel skeleton }} * 100
$$




\section{Results}

\subsection{Vascular atlas}

The 2D-TOF MRA data of 21 male C57BL/6 wild-type mice was used for the development of the vascular atlas. The segmentation resulted in delineation of the main large vessels that were spatially reliable across the sample of 21 mice. Furthermore, the segmentation was sorted into arteries (fig.2) and veins/sinuses (fig.3) to have a clear description of the vascular architecture (further described below). Given the limited spatial resolution of in-vivo MRA as well as the inter-subject small variability in vessel positions, the vessel sizes were substantially overestimated. To overcome this limitation, centerlines of the blood vessels were extracted by performing a skeletonization on the segmented data (fig.4). These centerlines depict the vascular atlas in single voxel lines representing the inner core of the blood vessels, which is also the position of highest inter-subject spatial reliability. Given this property, such an atlas representation is especially useful for investigating vascular effects of specific vessels in imaging data. Moreover, the skeletonization also allowed the development of a vascular graph, which represents the hierarchical structure of the vasculature and allows more explicit types of analyses, such as intervascular distance between vessels (or vessels and voxels), branching order, parent-child relationships, et cetera.

\subsubsection{Arteries}

The major arterial system of the mouse brain is visually depicted in figure $2 \mathrm{~A}$ and its hierarchical graph (fig. 2B) gives an overview of its connections. The blood supply to the mouse brain is provided via four main arteries i.e. (i) the two vertebral arteries that mainly supply blood to the arteries of the brain stem and the cerebellum and (ii) the two internal 
267 carotid arteries that mainly supply blood to arteries feeding the subcortical and cortical brain

268 regions.

269 The vertebral artery depicted one clear branch giving rise to the para-olivary artery

270 providing blood towards the inferior olive region. The two vertebral arteries then merge in

271 the midline into the basilar artery that is located on the ventral surface of the brainstem until

272 it reaches the pons/midbrain junction to give rise to two superior cerebral arteries. These

273 superior cerebral arteries end up in the lateral and medial superior cerebellar artery.

274 The internal carotid artery branches in rostral, lateral and caudal direction resulting in the

275 anterior, middle and posterior cerebral artery respectively. The anterior cerebral artery

276 of both internal carotid arteries heads rostrally on the ventral part of the brain and merges

277 together medially to form the azygos of the anterior cerebral artery. This artery is directed

278 dorsally and provides blood to the frontal lobe of the brain, including the olfactory bulb and

279 orbitofrontal regions by branching into the lateral orbitofrontal artery and later the medial

280 orbitofrontal artery. Next, the azygos of the anterior cerebral artery curves rostral and

281 gives rise to the azygos pericallosal artery that runs over the dorsal part of the corpus

282 callosum delivering blood to posterior-medial parts of the frontal-, parietal and temporal

283 lobes. The middle cerebral artery branches off from the lateral side of the internal carotid

284 artery and extends over the surface of the ventral part of the brain where it feeds blood to the

285 hypothalamus, caudate putamen and amygdala. It then continues in the dorsal direction on

286 the surface of the brain to provide blood to most of the cerebral cortex. The posterior

287 cerebral artery is dorsally directed where it is providing blood towards the caudal part of

288 the cerebrum including the hippocampus and the superior colliculus as well as cortical areas. 
289 Furthermore, the posterior cerebral artery connects with the superior cerebral artery via the posterior communicating artery.

291 At the ventral part of the brain around the level of the optic chiasm and the hypothalamus,

292 the arterial system forms a circulatory anastomosis called the circle of Willis (COW). This

293 anastomosis connects the internal carotid artery and the vertebral artery. The circle of Willis

294 is built from the basilar artery, which connects to both superior cerebral arteries that are

295 both connected with the posterior cerebral arteries via the posterior communicating

artery. The two posterior cerebral arteries are each connected with an internal carotid

artery that gives rise to the medial cerebral artery and the anterior cerebral artery. The anterior cerebral artery of both sides fuse into a circular anastomosis.

\subsubsection{Veins and Sinuses}

300 The major mouse-brain venous system produces a drainage system to remove blood 301 containing low nutrients as well as waste metabolites from the brain. The 2D-TOF MRA 302 detected the dorsal venous system as well as the deep venous system. Furthermore, veins 303 outside of the brain were observed and labeled. The venous system of the mouse brain is 304 visualized in figure 3A and its venous graph (fig. 3B) gives an overview of the connections 305 of each vein.

306 The detected dorsal venous system contains mainly four sinuses and their connecting veins.

307 The most rostral detected sinus is the (i) superior olfactory sinus which is connected in the 308 caudal direction with the (ii) superior sagittal sinus. These sinuses are located dorsally on

309 the pial surface and cover the midline of the cerebral cortex brain. In the rostral end, the 310 superior sagittal sinus produces the rostral rhinal veins in the ventral direction that reaches 
311 the frontal lobe. At the caudal end, the superior sagittal sinus splits into two lateral-ventral

312 oriented (iii) transverse sinuses that are each connected with a caudal medial oriented (iv)

313 sigmoid sinus. The aforementioned sinuses cover most of the pial surface of the cerebral

314 cortex. The transverse sinus was observed to have three branches i.e. a ventral directed

315 medial collicular vein that penetrates into the cerebral tissue to reach the colliculus areas,

316 the parafloccular vein that is directed caudally and reaches the paraflocculus, and finally

317 the rostral directed caudal rhinal vein.

318 The deep venous system branches off from the medial-dorsal part of the transverse sinus and

319 gives rise to the straight sinus. The sinus is rostroventrally directed and gives rise to the

320 inferior sagittal sinus as well as the vein of Galen. The vein of Galen was then observed to

321 produce two lateral oriented longitudinal hippocampal veins and further caudally two

322 thalamostriatal veins.

323 The venous blood is removed from the brain via venous outflow systems. Here the 2D-TOF

324 MRA detected the dorsal and caudal outflow. The dorsal outflow starts from the nasal

325 emissary vein, which is connected with the rostral part of the superior sagittal sinus. This

326 vein is oriented laterally and merges together with the supraorbital vein coming from the

327 eyes into the superior temporal vein. The caudal outflow system occurs from the

328 retroglenoid vein that is connected to the transverse sinus. The retroglenoid vein and the

329 superior temporal vein merges into the posterior facial vein that will eventually remove the

330 venous blood from the head via the external jugular vein.

\subsubsection{Skeletonization}


332 The developed 2D-TOF MRA atlas overestimates the vessel size. This is especially clear

333 when comparing the anatomical background images, where vessels produce hypo-intense

334 signals, with the 2D-TOF MRA data (fig. 4B). To overcome this, the vascular atlas was

335 further processed to only visualize the centerlines of each vessel using a skeletonization

336 process. By doing so, the whole brain vasculature could be represented by nodes and edges

337 model or with center lines with a width of one voxel (fig.4). Furthermore, this skeletonization

338 process allows for the development of a vascular graph model (see fig. 2B, 3B), visualizing

339 the hierarchical structure of the vascular architecture.

\subsection{Resting state functional connectivity}

341 Functional MRI sequences that rely upon the detection of the BOLD contrast such as gradient

342 echo EPI, are known to suffer from vascular influences especially due to large vessels. In this

343 study, rsfMRI data from a subset of animals used to build the reported atlas, were used to

344 investigate the extent of overlap between RSNs and the major mouse brain vasculature. The

345 RSNs were extracted from functional MRI data using independent component analysis (ICA)

346 and revealed nine functionally relevant networks (suppl. fig. 1). To calculate the percentage

347 overlap between the vasculature and the identified RSN components, we used the

348 skeletonized version of our atlas. Given that the skeletonized atlas has a single voxel

349 resolution, the overlap essentially finds voxels of the resting state network in which major

350 blood vessels are present. The results indicated that a number of networks demonstrated

351 substantial fraction of major vascular overlap (suppl. table 1). Specifically, the VOF values

352 were as follows sorted from highest to lowest overlap: Cingulate-Retrosplenial $\mathrm{I}=0.06$,

353 Cingulate - Retrosplenial II $=0.0483$, Orbitofrontal $=0.0428$, Hippocampal $=0.0417$, 
354 Striatum $=0.024$, Somatosensory barrel field $=0.0239$, Somatosensory - Motor $=0.0184$,

355 Primary somatosensory $=0.0183$, Visual Cortex $=0.0167$. The two networks with the highest

356 VOF were both networks which include the cingulate and retrosplenial cortex i.e. cingulate-

357 retrosplenial (Cg-Rs) I and II (fig. 5). Specifically, Cg-Rs I and Cg-Rs II components

358 demonstrated values of around $6 \%$ and $5 \%$ of voxels with overlap with major mouse

359 vasculature respectively. The $\mathrm{Cg}$-Rs I network was observed to include the complete

360 cingulate cortex and partially the retrosplenial cortex as well as motor cortex, preoptic area,

361 dorsal thalamus, superior colliculus and hippocampus. The Cg-Rs II network contained only

362 the posterior part of the cingulate, the entire retrosplenial cortex as well as the parietal

363 association and visual cortex. The substantial overlap of these networks with the major mouse

364 vasculature can directly be appreciated on the 3D visual representation of these components

365 as shown in figure 6 (fig. 6). Another two networks demonstrated substantial vascular overlap

366 of over $4 \%$ of voxels was the orbitofrontal and hippocampal networks, while all other

367 networks demonstrated vascular overlap values $<2.5 \%$.

368 To better understand the contribution of the arterial and venous/sinous compartments as well

369 as the contribution of specific vessels within each of the two cingulate - retrosplenial

370 networks, we also calculated the partial VOF (pVOF) for each vessel (table 1).

371 The Cg-Rs I network includes arteries, veins and sinuses while Cg-Rs II contained mainly

372 veins and sinuses. In the Cg-Rs I, a venous overlap could be seen from the superior sagittal,

373 transverse and the straight sinuses including all its labeled branches. The arterial overlap of

374 the Cg-Rs I involved the internal carotid artery, anterior cerebral artery and its branches, the

375 posterior/middle cerebral artery and the superior cerebellar artery. The Cg-Rs II vascular 
376 overlap contained mainly venules i.e. the superior sagittal sinus and the straight sinus and its

377 branches. Arterial overlap was limited to the superior/middle cerebral artery, azygos

378 pericallosal artery and the medial orbitofrontal artery.

\section{4. Discussion}

380 An intact vascular architecture is essential for the healthy function of the brain. It is therefore

381 of utmost importance for many studies to be able to evaluate the contribution of vessel signals

382 and/or detect vascular abnormalities. Previously developed mouse cerebral vascular atlases

383 mainly focused on the visualization of the - as much as possible - complete vascular tree

384 down to the small vessels and capillaries. This resulted in high-resolution large datasets that

385 tend to be time consuming to acquire and difficult to process [31] and therefore report results

386 from a limited number of animals $[17,18]$. In the current study, we developed of a mouse

387 cerebral vascular atlas that focusses on visualizing the spatially reliable larger vessels in a

388 high number of $\mathrm{C} 57 \mathrm{BL} / 6$ wild-type mice $(\mathrm{N}=21)$ at the age of 12 weeks. In contrast to

389 previous atlases, this atlas was specifically developed to be used as a reference vascular atlas

390 for group analysis across studies that use C57BL/6 wild-type mice. By using high number of

391 animals, the averaging of the individual cerebral vasculature enhances spatial reliable vessels

392 and suppresses vessels demonstrate anatomical variations across subjects. In addition, the

393 vascular atlas is co-registered to high-resolution anatomical data which is adding spatial

394 information to the vasculature. 
396 The vascular atlas was developed using a 2D-TOF MRA sequence. This MRI sequence was

397 specifically chosen for its high sensitivity towards the vasculature and its complete non-

398 invasiveness, which allows for easy implementation in future MRI studies. The method

399 allowed for the detection of blood vessels based upon their flow perpendicular to the slice

400 position. It was therefore required to acquire three orthogonally positioned 2D-TOF MRA

401 scans to obtain the complete overview of the vasculature. The 2D-TOF MRA was chosen

402 instead of its 3D-TOF MRA counterpart due to its superior sensitivity to slow flow allowing

403 for the detection of a wider range of vessels. Moreover, 3D-TOF MRA is also more sensitive

404 to motion artifacts that could compromise the data acquisition. By combining three 2D-TOF

405 MRA datasets, we implemented a method for non-invasive reproducible detection of in-vivo

406 cerebral vasculature in mice.

\subsection{Vascular atlas}

408

409

410

411

412

The presented in-vivo vascular atlas contains multiple datasets: i) a cerebral vascular atlas;

ii) a digital segmentation of large vessels (arteries and veins/sinuses); iii) a high resolution T2 anatomical background; iv) a skeletonized vascular atlas; and v) a vascular graph model.

The cerebral vascular atlas is an average vascular template created by all co-registered 2DTOF MRA datasets and is accompanied by a T2-weighted anatomical template. These datasets can be used as a co-registration endpoint to acquire either vascular information or anatomical information. The templates will be especially useful for studies that acquire 3D anatomical information (MRI, CT) and want to extract cerebral vascular information or for studies that rely on the acquisition of vascular information (fUS, IOS) and want to get anatomical information. The vascular atlas was manually segmented, which resulted in the 
418 labeling of spatially reliable vessels. The segmentation included the main large arteries, veins

419 and sinuses within the brain as well as their outflow systems going around the head. However,

420 small branches were not included due to spatial variation between subjects. The anatomical

421 structure of the vessels was similar as in the paper of Xiong et al. that produced a high

422 resolution ex-vivo vascular atlas in the same C57BL/6 mouse strain [18]. Small differences

423 were found between our labeling and the vascular atlas of Dorr et al. that mainly revolved

424 around the posterior communicating artery being connected with the posterior cerebral and

425 internal carotid artery, while in our dataset it was connecting the posterior and superior

426 cerebral artery [17]. However, this can be explained as the aforementioned atlas was acquired

427 in a different mouse strain i.e. CBA mouse strain. Previous studies have reported structural

428 cerebral vascular differences between mouse strains [32, 34, 35]. This indicates caution when

429 using the vascular atlas in other strains than the C57BL/6 strain. Furthermore, due to the

430 spatial variability of the cerebral vasculature, the segmentation of the blood vessels were

431 overestimated. To overcome this, the segmentation was skeletonized and vascular centerlines

432 were extracted. As the vascular centerlines depicts blood vessels as single voxel lines it is a

433 more appropriate tool to investigate the spatial location of the vasculature. The centerlines

434 will therefore be of use in studies that are particularly interested in defining the presence of

435 vasculature in their datasets. In addition to the centerlines, the skeletonization process results

436 in a representation of the vasculature as nodes and edges allowing for the development of a

437 vascular graph model. The vascular graph model contains the hierarchical structure of the

438 cerebral vascular architecture and allowed automatic clustering of the arterial and venous

439 system. This model can be used to get insights in blood flow patterns which could be valuable

440 for studies performing BOLD and perfusion analysis. 


\subsection{Application of the vascular atlas in rsfMRI}

442 RsfMRI relies upon the detection of fluctuations in the BOLD signal, which is assumed to

443 be an indirect measurement of changes in neural activity detected via the neurovascular

444 coupling. As BOLD signals are based on detecting changes in blood oxygenation, it is

445 assumed that it is most sensitive to the capillaries where most of the oxygenation exchange

446 occurs. However, the BOLD signal has been shown to be influenced by the macrovasculature

447 [22]. More specifically, confounding BOLD signals of vascular origin have been widely

448 detected coming from downstream draining veins [22]. This is caused due to the

449 accumulation of the blood coming from the capillaries to the draining veins and thus

450 smearing out the BOLD signal via the venules. Therefore, as an application of the vasculature

451 atlas, we implemented the atlas to rsfMRI data to investigate the vascular contribution in the

452 observed RSN. The vascular overlap analysis could show that the two highest overlapping

453 components were components covering connections between the cingulate and retrosplenial

454 cortex (Cg-Rs I and II). These regions are commonly part of the default mode-like network

455 in mice $[27,29,36]$. Using the vascular atlas, we could reveal that the Cg-Rs I \& II RSNs

456 have the strongest vascular contribution, but this is still limited to around $6 \%$ and $5 \%$ of the

457 original resolution EPI voxels being penetrated by the large, reliable vessels included in our

458 atlas. This indicates that even if these networks suffer from slightly higher vascular

459 influences in comparison to some other networks, the majority of their voxels are not

460 penetrated by the large vessels and thus their functional correlations most probably reflect

461 neuronal components. 


\section{Conclusion}

464 In conclusion, this article presents a newly developed in-vivo mouse brain vascular atlas

465 based on 21 adult male C57BL/6 wild-type mice (12 weeks old) acquired with MRI. The

466 atlas includes: i) a cerebral vascular atlas; ii) segmentation of large vessels; iii) a high

467 resolution T2 anatomical background; iv) a skeletonized vascular atlas; and v) a vascular

468 graph model that are available online for download. The atlas acts as a co-registration

469 template for studies that require both vascular and anatomical information. As a potential

470 application, we superimposed the atlas on rsfMRI data of adult male C57BL/6 wild-type

471 mice which enabled us to identify a differential degree of overlap between the vasculature

472 and the different RSNs. Undoubtedly the use of this atlas will contribute to a more solid

473 interpretation and post processing of resting state data obtained in mice.

\section{Acknowledgments}

475 This research was supported by the fund of Scientific Research Flanders (grant agreement 476 G048917N), Flagship ERA-NET (FLAG-ERA) FUSIMICE (grant agreement G.0D7651N),

477 , Molecular Imaging of Brain Pathophysiology (BRAINPATH) under Grant Agreement

478 Number 612360 within the Marie Curie Actions-Industry-Academia Partnerships and

479 Pathways (IAPP) program. MRI equipment was funded by the Flemish Impulse funding for

480 heavy scientific equipment (granted to AVdL). The computational resources and services

481 used in this work were provided by the HPC core facility CalcUA of the Universiteit

482 Antwerpen, the VSC (Flemish Supercomputer Center), funded by the Hercules Foundation 483 and the Flemish Government - department EWI. 


\section{Author contribution statement}

485 Rukun Hinz: Study initiation, Data acquisition, Data processing, Writing of the article

486 Meriam Malekzadeh: Data processing: vascular segmentation

487 Lore M. Peeters: Study initiation, Data processing, Writing of the article

488 Verdi Vanreusel: Data processing: Co-registration

489 Marleen Verhoye: Study initiation, Writing of the article

490 Annemie Van der Linden: Study initiation, Writing of the article

491 Georgios A. Keliris: Study initiation, Data Processing, Writing of the article, Study

492 supervision

493

494 Disclosure/conflict of interest

495 The Author(s) declare(s) that there is no conflict of interest 
496

497

498

499

500

501

502

503

504

505

506

507

508

509

510

511

512

513

514

515

\section{References}

[1] Mergenthaler P, Lindauer U, Dienel GA, et al. Sugar for the brain: the role of glucose in physiological and pathological brain function. Trends Neurosci 2013; 36: $587-97$.

[2] Jain V, Langham MC, Wehrli FW. MRI estimation of global brain oxygen consumption rate. J Cereb Blood Flow Metab 2010; 30: 1598-607.

[3] Govindpani K, McNamara LG, Smith NR, et al. Vascular Dysfunction in Alzheimer's Disease: A Prelude to the Pathological Process or a Consequence of It? J Clin Med; 8. Epub ahead of print 10 May 2019. DOI: $10.3390 / j \mathrm{~cm} 8050651$.

[4] Lin C-Y, Hsu Y-H, Lin M-H, et al. Neurovascular abnormalities in humans and mice with Huntington's disease. Exp Neurol 2013; 250: 20-30.

[5] D'haeseleer M, Cambron M, Vanopdenbosch L, et al. Vascular aspects of multiple sclerosis. Lancet Neurol 2011; 10: 657-66.

[6] Amato SP, Pan F, Schwartz J, et al. Whole Brain Imaging with Serial Two-Photon Tomography. Front Neuroanat 2016; 10: 31.

[7] Mayerich D, Kwon J, Sung C, et al. Fast macro-scale transmission imaging of microvascular networks using KESM. Biomed Opt Express 2011; 2: 2888-96.

[8] Xue S, Gong H, Jiang T, et al. Indian-ink perfusion based method for reconstructing continuous vascular networks in whole mouse brain. PLoS One 2014; 9: e88067.

[9] Wu J, He Y, Yang Z, et al. 3D BrainCV: simultaneous visualization and analysis of 
cells and capillaries in a whole mouse brain with one-micron voxel resolution. Neuroimage 2014; 87: 199-208.

[10] Lugo-Hernandez E, Squire A, Hagemann N, et al. 3D visualization and quantification of microvessels in the whole ischemic mouse brain using solventbased clearing and light sheet microscopy. J Cereb Blood Flow Metab 2017; 37: $3355-3367$.

[11] Todorov MI, Paetzold JC, Schoppe O, et al. Machine learning analysis of whole mouse brain vasculature. Nat Methods 2020; 17: 442-449.

[12] Starosolski Z, Villamizar CA, Rendon D, et al. Ultra High-Resolution In vivo Computed Tomography Imaging of Mouse Cerebrovasculature Using a Long Circulating Blood Pool Contrast Agent. Sci Rep 2015; 5: 10178.

[13] Klohs J, Baltes C, Princz-Kranz F, et al. Contrast-Enhanced Magnetic Resonance Microangiography Reveals Remodeling of the Cerebral Microvasculature in Transgenic ArcA Mice. J Neurosci 2012; 32: 1705-1713.

[14] Tiran E, Ferrier J, Deffieux T, et al. Transcranial Functional Ultrasound Imaging in Freely Moving Awake Mice and Anesthetized Young Rats without Contrast Agent. Ultrasound Med Biol 2017; 43: 1679-1689.

[15] Quintana DD, Lewis SE, Anantula Y, et al. The cerebral angiome: High resolution MicroCT imaging of the whole brain cerebrovasculature in female and male mice. Neuroimage 2019; 202: 116109. 
neurovasculature with magnetic resonance microscopy. PLoS One 2011; 6: e22643.

538 [17] Dorr A, Sled JG, Kabani N. Three-dimensional cerebral vasculature of the CBA mouse brain: A magnetic resonance imaging and micro computed tomography study. Neuroimage 2007; 35: 1409-1423.

541 [18] Xiong B, Li A, Lou Y, et al. Precise Cerebral Vascular Atlas in Stereotaxic Coordinates of Whole Mouse Brain. Front Neuroanat 2017; 11: 128.

543 [19] Deffieux T, Demene C, Pernot M, et al. Functional ultrasound neuroimaging: a 544 review of the preclinical and clinical state of the art. Curr Opin Neurobiol 2018; 50: 128-135.

546 [20] Hillman EMC. Optical brain imaging in vivo: techniques and applications from animal to man. J Biomed Opt; 12: 051402.

548 [21] Hillman EMC. Coupling mechanism and significance of the BOLD signal: a status report. Annu Rev Neurosci 2014; 37: 161-81.

550 [22] Kennerley AJ, Mayhew JE, Redgrave P, et al. Vascular Origins of BOLD and CBV fMRI Signals: Statistical Mapping and Histological Sections Compared. Open Neuroimag J 2010; 4: 1-8.

553 [23] Turner R. How much cortex can a vein drain? Downstream dilution of activationrelated cerebral blood oxygenation changes. Neuroimage 2002; 16: 1062-7.

555 [24] Grandjean J, Canella C, Anckaerts C, et al. Common functional networks in the mouse brain revealed by multi-centre resting-state fMRI analysis. Neuroimage 2019; 
116278.

558 [25] Tong Y, Hocke LM, Frederick BB. Low Frequency Systemic Hemodynamic 'Noise'

559

560

561

562

563

564

565

566

567

568

569

570

571

572

573

574

575

576

577 in Resting State BOLD fMRI: Characteristics, Causes, Implications, Mitigation Strategies, and Applications. Front Neurosci 2019; 13: 787.

[26] Dorr AE, Lerch JP, Spring S, et al. High resolution three-dimensional brain atlas using an average magnetic resonance image of 40 adult $\mathrm{C} 57 \mathrm{Bl} / 6 \mathrm{~J}$ mice. Neuroimage 2008; 42: 60-69.

[27] Liska A, Galbusera A, Schwarz AJ, et al. Functional connectivity hubs of the mouse brain. Neuroimage 2015; 115: 281-291.

[28] Jonckers E, Van Auderkerke J, De Visscher G, et al. Functional connectivity fMRI of the rodent brain: comparison of functional connectivity networks in rat and mouse. PLoS One 2011; 6: e18876.

[29] Stafford JM, Jarrett BR, Miranda-Dominguez O, et al. Large-scale topology and the default mode network in the mouse connectome. Proc Natl Acad Sci 2014; 111 : $18745-18750$.

[30] Grandjean J, Canella C, Anckaerts C, et al. Common functional networks in the mouse brain revealed by multi-centre resting-state fMRI analysis. Neuroimage 2019; 205: 116278 .

[31] Hess A, Hinz R, Keliris GA, et al. On the Usage of Brain Atlases in Neuroimaging Research. Mol Imaging Biol; 20. Epub ahead of print 2018. DOI: 10.1007/s11307018-1259-y. 
578 [32] Ward R, Collins RL, Tanguay G, et al. A quantitative study of cerebrovascular $579 \quad$ variation in inbred mice. J Anat 1990; 173: 87-95.

580 [33] Barone FC, Knudsen DJ, Nelson AH, et al. Mouse Strain Differences in Susceptibility to Cerebral Ischemia are Related to Cerebral Vascular Anatomy. $J$

[34] Beckmann N. High resolution magnetic resonance angiography non-invasively reveals mouse strain differences in the cerebrovascular anatomy in vivo. Magn Reson Med 2000; 44: 252-8.

586

[36] Hinz R, Peeters LM, Shah D, et al. Bottom-up sensory processing can induce negative BOLD responses and reduce functional connectivity in nodes of the default mode-like network in rats. Neuroimage 2019; 197: 167-176. 
594 Figure legends

595 Figure 1. Time of flight magnetic resonance imaging. Example of a 2D-TOF MRA dataset of a

596 representative animal. Dataset included three orthogonally positioned 2D-TOF MRA scans with the

597 slice package placed (A) Coronally, (B) Axially and (C) Sagittally allowing the visualization of

598 vascular flow in the rostral-caudal, dorsal-ventral and left right direction respectively. Datasets were

599 combined using a maximum intensity projection (MIP) to visualize whole brain vasculature (D).

600 Figure 2. Arterial System and vascular graph. A. Three-dimensional overview of the arterial

601 system with annotations included in the vascular atlas. Crosshair indicates rostral-caudal (Ro-Ca),

602 dorsal-ventral (D-V) and left-right (L-R) direction. B. Hierarchical structure and annotations of the

603 mouse brain arterial system.

604 Figure 3. Venous System and vascular graph. A. Three-dimensional overview of the venous

605 system with annotations included in the vascular atlas. Crosshair indicates rostral-caudal (Ro-Ca),

606 dorsal-ventral (D-V) and left-right (L-R) direction. B. Hierarchical structure and annotations of the

607 mouse brain venous system.

608 Figure 4. Skeletonization of the mouse brain vasculature. A. The labeling of the vasculature was

609 skeletonized and is represented in nodes and edges. The nodes represent starting, end or bifurcation

610 points, while edges indicate vessel paths. Furthermore, the skeletonization process allows for fast

611 detection of arteries (red) and veins (blue) as both were detected as separate circuits B. When

612 comparing the labeled vasculature with the anatomical template, a discrepancy between vessel size

613 could be observed. Centerlines can be used to demonstrate the presence of a vessel irrespectively of

614 its size. For visualization purposes centerlines were depicted with a centerline thickness of 4 voxels.

615 Figure 5. Resting state networks with highest vascular overlap. Statistical map of two resting

616 state networks with highest vascular overlap (A) Cingulate-Retrosplenial I (0.21\%) and (B)II

$617(0.17 \%)$. Statistical maps were produced by one sample t-test $(\mathrm{p}<0.001$, uncorrected) performed on

618 the outcome of an independent component analysis. Color bar represents t-values.

619 Figure 6. Resting state networks and vascular overlap. 3D visual representation of the statistical

620 maps of (A) cingulate-retrosplenial I and (B) II network together with the brain vasculature (MIP).

621 Statistical maps were produced by one sample t-test $(\mathrm{p}<0.001$, uncorrected) performed on the

622 outcome of an independent component analysis. Color bar represents t-values. Blood vessel that

623 partially overlap with the resting state network were highlighted in green. 
bioRxiv preprint doi: https://doi.org/10.1101/2021.10.21.465264; this version posted October 22, 2021. The copyright holder for this preprint (which was not certified by peer review) is the author/funder, who has granted bioRxiv a license to display the preprint in perpetuity. It is made available under aCC-BY-NC-ND 4.0 International license.

624 Table 1. Percent Vascular Inclusion (PVI) in Cingulate-Retrosplenial I and II. Percentage

625 vessel of the vascular skeleton contained within the resting state component.

626 


\begin{tabular}{|c|c|c|}
\hline Vessel Name & $\begin{array}{c}\text { Cingulate - Retrosplenial } \\
\text { I } \\
\text { (\% vessel within } \\
\text { component) }\end{array}$ & $\begin{array}{c}\text { Cingulate - Retrosplenial } \\
\text { II } \\
\text { (\% vessel within } \\
\text { component) }\end{array}$ \\
\hline Internal Carotid Artery (Right) & 26.67 & $\bar{l}$ \\
\hline Internal Carotid Artery (Left) & 28.57 & / \\
\hline Superior Cerebral Artery (Right) & 15.38 & 5.13 \\
\hline $\begin{array}{l}\text { Lateral Superior Cerebellar Artery } \\
\text { (Right) }\end{array}$ & 78.57 & / \\
\hline $\begin{array}{l}\text { Lateral Superior Cerebellar Artery } \\
\text { (Left) }\end{array}$ & 52.38 & / \\
\hline $\begin{array}{l}\text { Posterior Communicating Artery } \\
\text { (Right) }\end{array}$ & 8.33 & / \\
\hline Posterior Cerebral Artery (Right) & 25.00 & / \\
\hline Posterior Cerebral Artery (Left) & 21.57 & / \\
\hline Middle Cerebral Artery (Right) & 11.59 & 10.14 \\
\hline Middle Cerebral Artery (Left) & 5.63 & 5.63 \\
\hline Anterior Cerebral Artery (Right) & 64.29 & / \\
\hline Anterior Cerebral Artery (Left) & 31.03 & / \\
\hline Azygos of Anterior Cerebral Artery & 27.27 & / \\
\hline Azygos Pericallosal Artery & 41.82 & 50.91 \\
\hline Medial Orbitofrontal Artery & 14.29 & 2.86 \\
\hline Superior Sagittal Sinus & 13.58 & 24.53 \\
\hline Transverse Sinus (Right) & / & / \\
\hline Transverse Sinus (Left) & 22.73 & / \\
\hline Medial Collicular Vein (Left) & 7.41 & / \\
\hline Straight Sinus & 100.00 & 92.31 \\
\hline Inferior Sagittal Sinus & 100.00 & 100.00 \\
\hline Vein of Galen & 79.17 & 100.00 \\
\hline $\begin{array}{l}\text { Longitudinal Hippocampal Vein } \\
\text { (Right) }\end{array}$ & 100.00 & 100.00 \\
\hline Longitudinal Hippocampal Vein (Left) & 100.00 & 41.67 \\
\hline Thalamostriate Vein_(Right) & 50,00 & 3.85 \\
\hline Thalamostriate vein (Left) & 50.00 & 10.00 \\
\hline
\end{tabular}




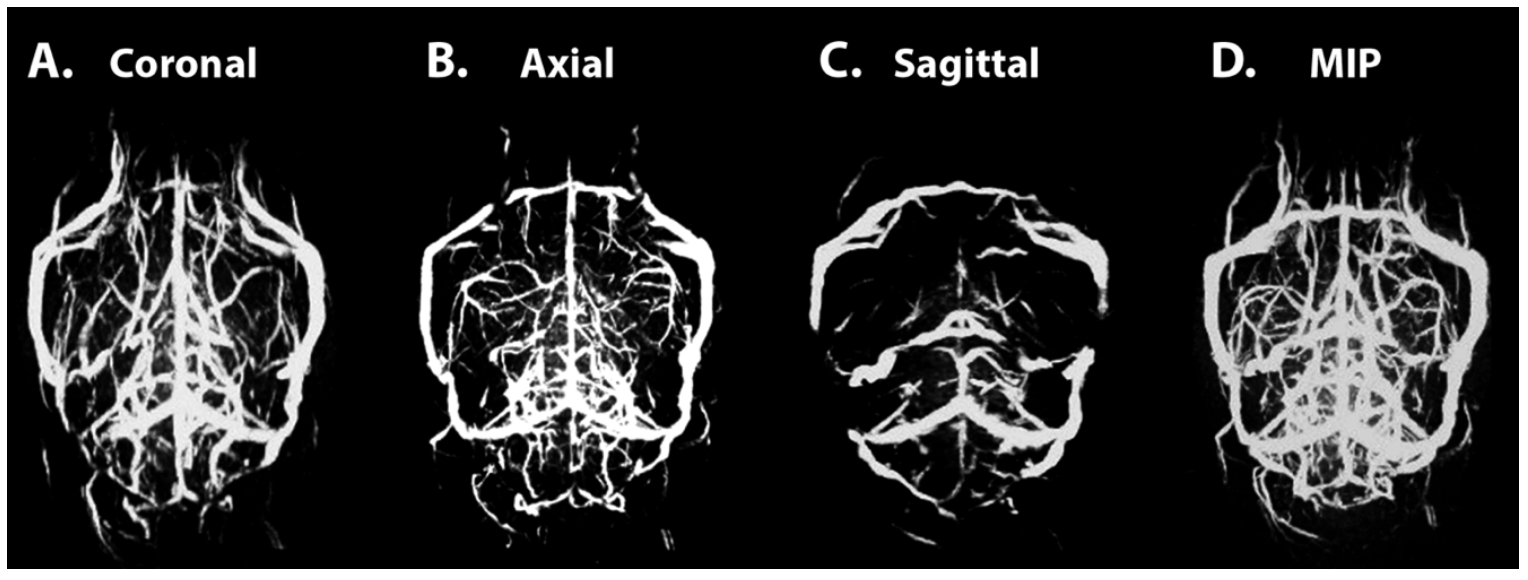

631 Figure 1. Time of flight magnetic resonance imaging. Example of a 2D-TOF MRA dataset of a

632 representative animal. Dataset included three orthogonally positioned 2D-TOF MRA scans with the

633 slice package placed (A) Coronally, (B) Axially and (C) Sagittally allowing the visualization of

634 vascular flow in the rostral-caudal, dorsal-ventral and left right direction respectively. Datasets were

635 combined using a maximum intensity projection (MIP) to visualize whole brain vasculature (D). 
A.
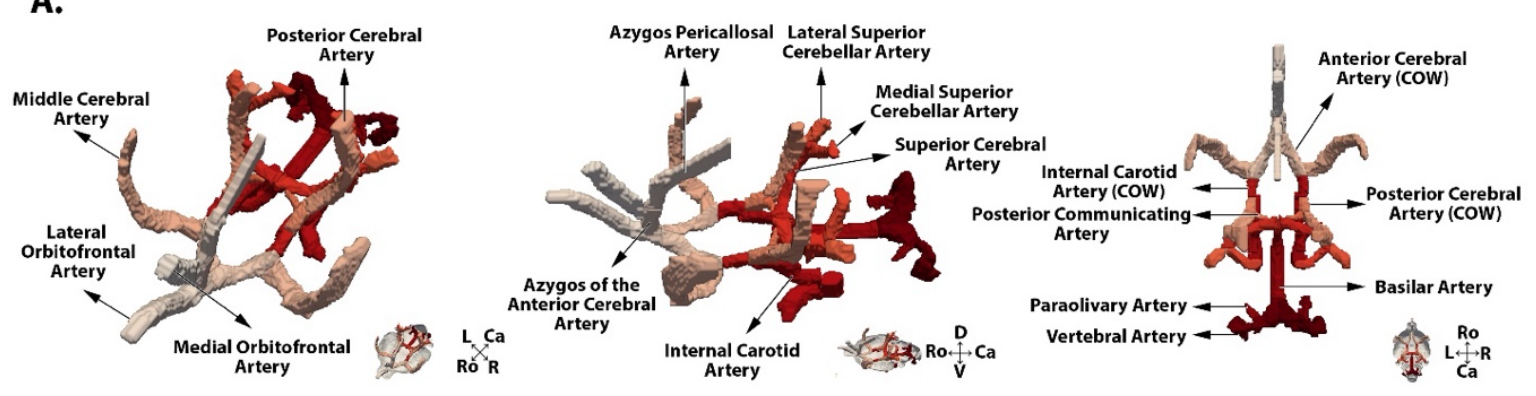

B.
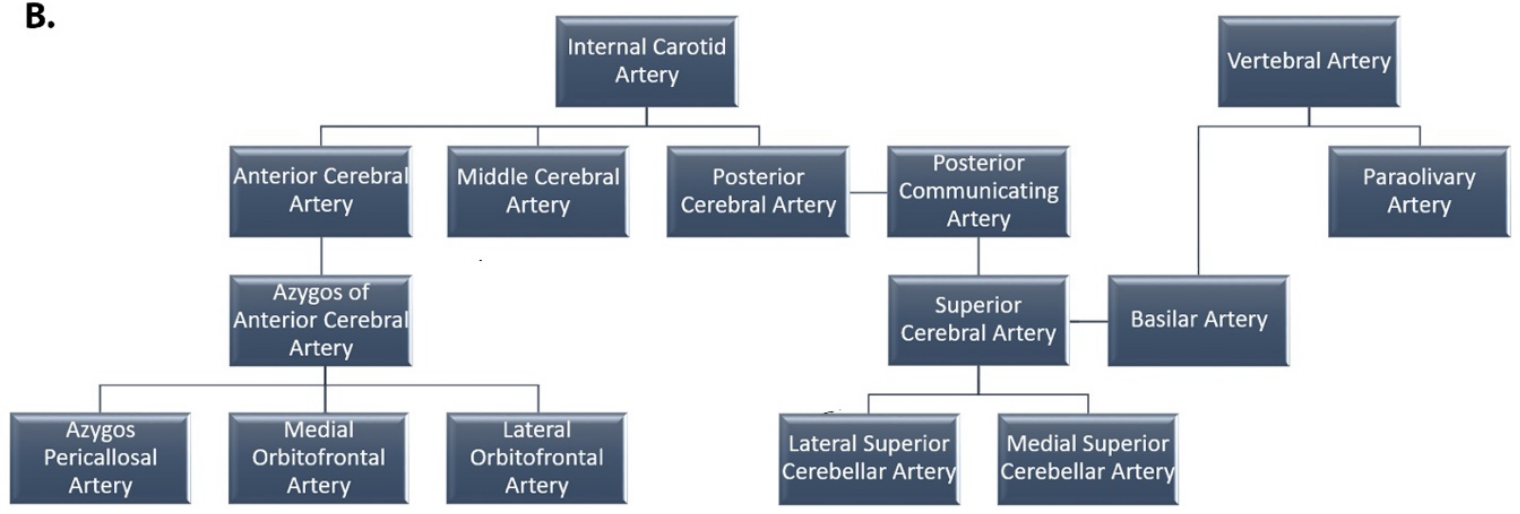
Artery

638 Figure 2. Arterial System and vascular graph. A. Three-dimensional overview of the arterial

639 system with annotations included in the vascular atlas. Crosshair indicates rostral-caudal (Ro-Ca),

640 dorsal-ventral (D-V) and left-right (L-R) direction. B. Hierarchical structure and annotations of the 641 mouse brain arterial system. 


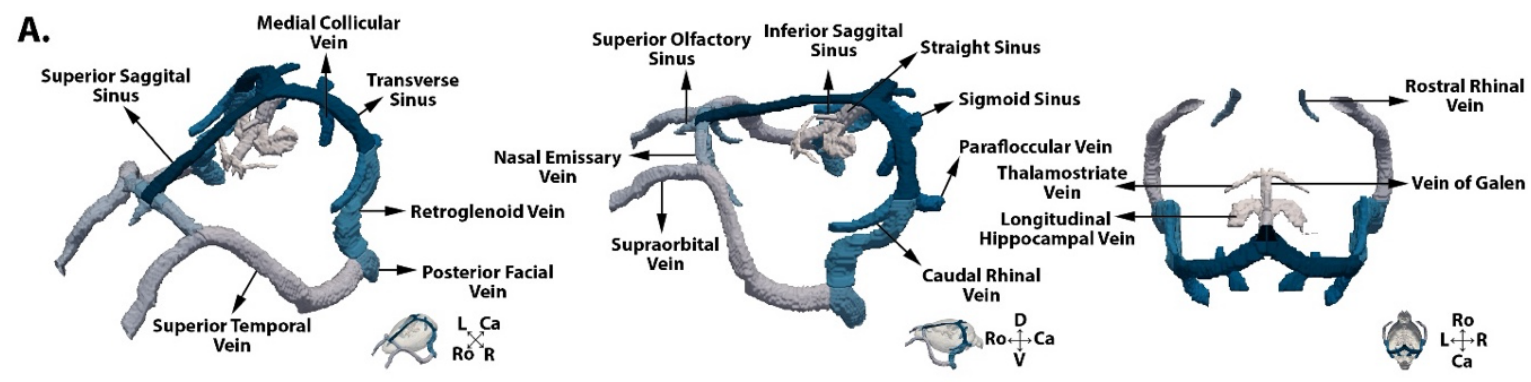

B.

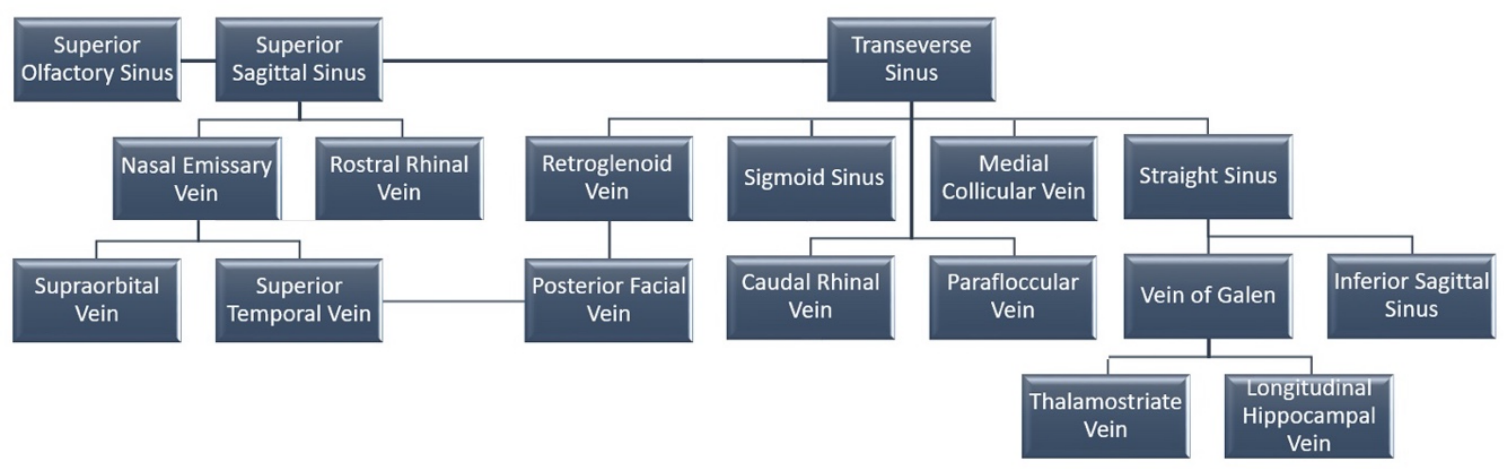

644 Figure 3. Venous System and vascular graph. A. Three-dimensional overview of the venous

645 system with annotations included in the vascular atlas. Crosshair indicates rostral-caudal (Ro-Ca),

646 dorsal-ventral (D-V) and left-right (L-R) direction. B. Hierarchical structure and annotations of the 647 mouse brain venous system. 
A.
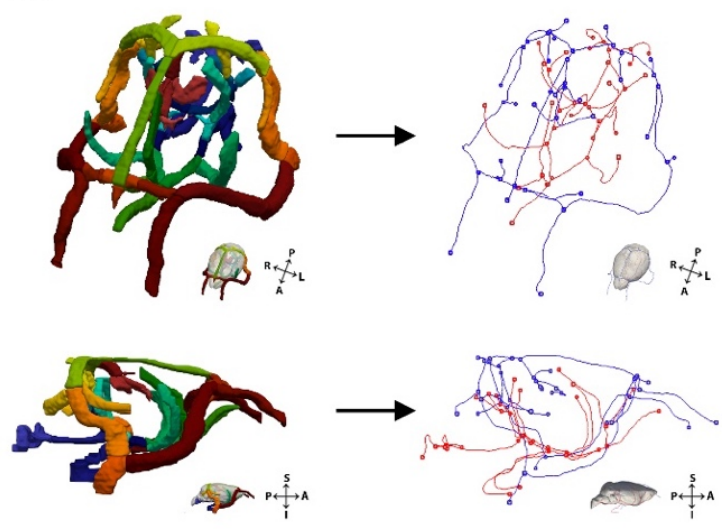

B.

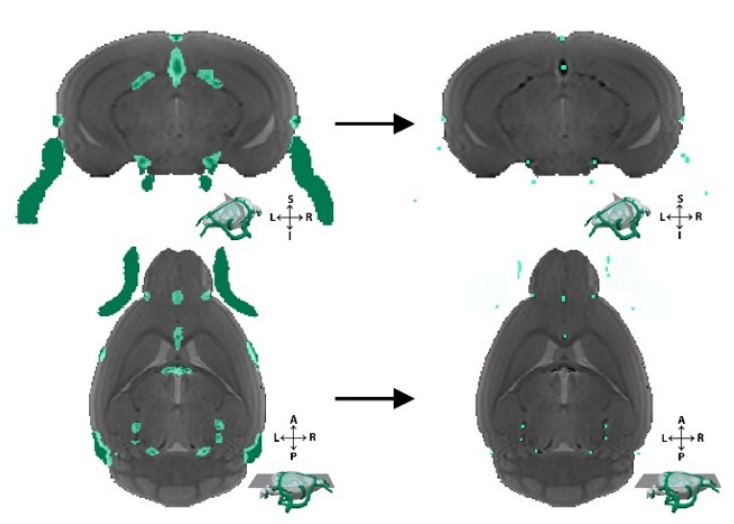

Figure 4. Skeletonization of the mouse brain vasculature. A. The labeling of the vasculature was skeletonized and is represented in nodes and edges. The nodes represent starting, end or bifurcation points, while edges indicate vessel paths. Furthermore, the skeletonization process allows for fast detection of arteries (red) and veins (blue) as both were detected as separate circuits B. When comparing the labeled vasculature with the anatomical template, a discrepancy between vessel size could be observed. Centerlines can be used to demonstrate the presence of a vessel irrespectively of its size. For visualization purposes centerlines were depicted with a centerline thickness of 4 voxels. 


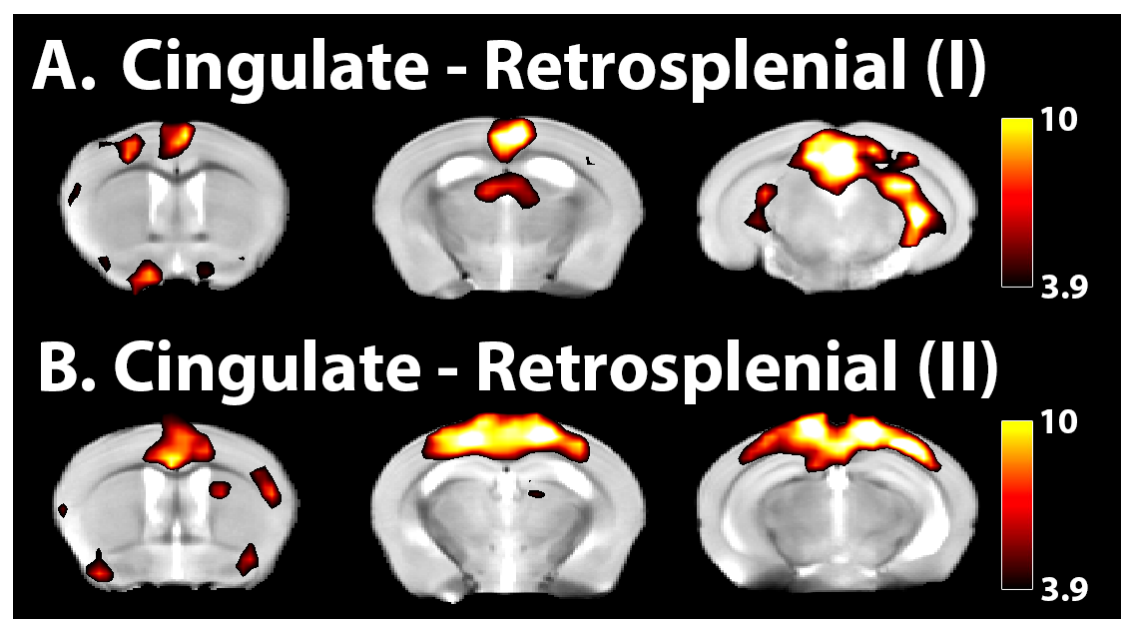

659 Figure 5. Resting state networks with highest vascular overlap. Statistical map of two resting 660 state networks with highest vascular overlap (A) Cingulate-Retrosplenial I (0.21\%) and (B)II

661 (0.17\%). Statistical maps were produced by one sample t-test $(\mathrm{p}<0.001$, uncorrected) performed on 662 the outcome of an independent component analysis. Color bar represents t-values. 

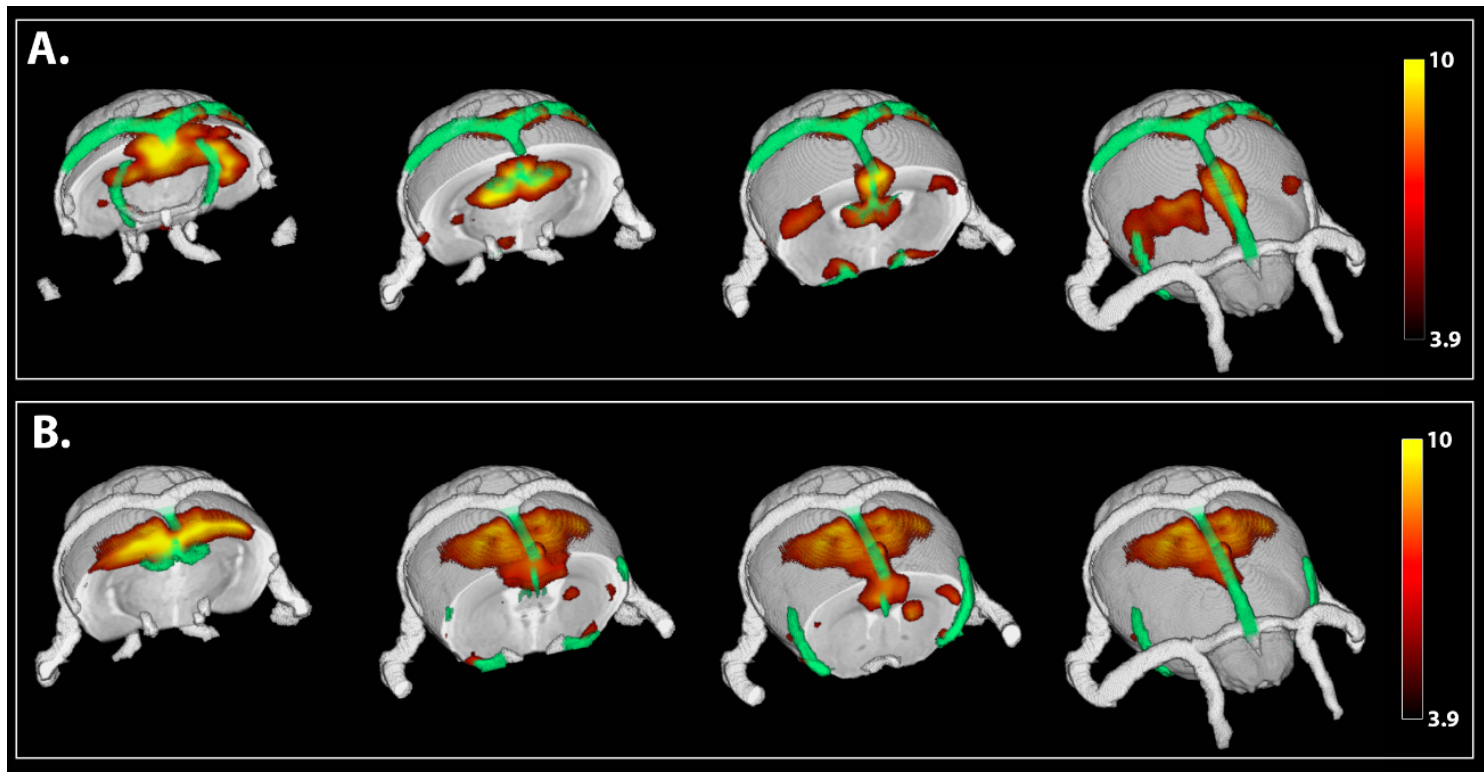

665 Figure 6. Resting state networks and vascular overlap. 3D visual representation of the statistical

666 maps of (A) cingulate-retrosplenial I and (B) II network together with the brain vasculature (MIP).

667 Statistical maps were produced by one sample t-test ( $<<0.001$, uncorrected) performed on the

668 outcome of an independent component analysis. Color bar represents t-values. Blood vessel that

669 partially overlap with the resting state network were highlighted in green. 